\title{
Factors required for mobile search going mainstream
}

José Luis Gómez-Barroso

\author{
Margherita Bacigalupo, Stavri G. Nikolov and Ramón Compañó
}

\author{
Claudio Feiióo
}

\begin{abstract}
Purpose - This article aims to review the technological and socio-economic conditions which will influence the development of the mobile search market.

Design/methodology/approach - An expert workshop with academics, industry representatives and market analysts was organised to discuss and analyse the results of an online survey of techno-economic and socio-economic aspects concerning the evolution of mobile search.

Findings - Despite clear positive signs, forecasted great expectations around mobile search are not yet supported by economic market evidence. Substantial development work for creating new applications ripe for the mass market is still needed, although there seems to be no fundamental technological barrier to that. Location-based services, augmented reality, real-time information search, and social network search and recommendations, have been identified as some of the key trends that may shape the future of mobile search. User demand for innovative mobile search-based applications is largely taken for granted, but experts lack a clear view on suitable business models that would allow for sustainable economic development.

Originality/value - Innovating is not only inventing. Understanding what drives customers' willingness to use - and to pay for - a product or service is essential in order to design appropriate services and introduce these innovations to the market. There is a growing literature on mobile search-related technology, but the market context is largely unexplored.
\end{abstract}

\section{Introduction}

It was an open secret: the internet was going mobile, and nobody doubted it. Yet without official data, nobody knew when and to what extent. Consultancy firms regularly released market figures and forecasts, frequently accompanied by authoritative headlines, but often obtained by unreliable or obscure procedures. It was not until December 2010 that the Organisation for Economic Co-operation and Development (OECD) released new statistics on broadband penetration in OECD countries, reporting for the first time a wireless broadband penetration indicator (Organisation for Economic Co-operation and Development, 2010). In June 2010, 294 million fixed broadband subscriptions were counted in the OECD area. In contrast, the 
number of mobile broadband subscriptions (those included in a mobile voice plan) and dedicated mobile data subscriptions (which need an additional data plan) amounted to 435 million, i.e. 1.5 times the number of fixed broadband connections. Korea (95.0), Sweden (75.6), Japan (75.3) and Norway (72.8) show the highest wireless broadband penetration in terms of subscribers per 100 inhabitants. Of the 29 countries for which there is data, 11 had penetration higher than 40 percent and 17 countries over 30 percent. The leaders in fixed broadband - The Netherlands, Denmark and Switzerland - only account for around 37 wired broadband subscriptions per 100 inhabitants. It is not meaningful to directly compare the numbers, as fixed broadband plans are usually acquired for a household, which usually has more than one person, but nevertheless they are eloquent enough.

Adding to these reliable OECD data, a likely scenario can be completed by making use of data - never completely accurate but significant enough - released by consultancy firms. According to Gartner (2010) worldwide smartphone sales accounted for 19.3 percent of overall mobile phone sales in the third quarter of 2010 , growing 96 percent from the third quarter of 2009. Nielsen (2011) reports that, as of November 2010, 31 percent of US mobile subscribers had smartphones (45 percent among those who have acquired a new cellphone in the previous six months) up from 24 percent on June. Signs of change are complemented by firms in the sector. When reporting its results for October 2010, Mixi, Japan's leading social network, disclosed the number of total monthly page views to be 31.2 billion, of which 26.1 billion were accessed via mobiles, remarkably almost 84 percent (Mixi, 2010).

How is mobile internet taking shape? Similarly to its wired counterpart, wireless internet users will continue to demand access to data and content in an efficient and user-friendly manner. Assuming the lessons of the fixed internet experience hold, search engines are called to play a dominant role in configuring mobile internet markets. Consequently the mobile search market is regarded as a major business opportunity in the ICT sector. Moreover the field is certainly large enough even for newer or smaller competitors (Gómez-Barroso et al., 2011b).

Notwithstanding these commonly accepted promising prospects, the mobile search market remains below the expectations forecasted some years ago. Today its evolution remains difficult to predict and its real impact difficult to quantify. This is due to a lack of understanding of the factors governing a successful deployment. Generally these factors are presented as an extended list of technological drivers and constraints, with hardly any mention of related socio-economic factors, thus hindering the proper discernment of the barriers lying ahead.

This paper aims to narrow this knowledge gap by synthesising a consultation process with reputable experts on the trends, drivers and bottlenecks affecting the future development of mobile search. As a final step of this process, an expert workshop was organised, as part of a conference. Its aim was to agree upon the techno-economic and socio-economic trends that are currently shaping the evolution of the mobile search market. In particular the workshop focused on search services arising from the unique features of mobile devices and mobile environments, e.g. context-aware or location-based services, and social (network) search.

The paper first describes the study's methodology. It is followed by a discussion of the market dynamics of mobile search. The barriers - of regulatory, technical, 
economic, or social nature - hampering and the challenges influencing the mobile search markets are then presented. The paper ends with some conclusions.

\section{Methodology}

The methodology followed a two-step Delphi-type approach, by which the results of an anonymous online survey (first step) was subsequently discussed in a face-to-face workshop (second step). The first step was carried out in April 2010. The authors designed a questionnaire taking into account the experience of a questionnaire used for a previous study (Gómez-Barroso et al., 2010). The questionnaire included a number of control questions in order to check the consistency with the previous survey. The questionnaire was sent to 150 hand-picked professionals in the field. The typical profile of survey addressees were experts with at least five years of professional experience in the field in decision making positions, either as researchers or managers. The sample population was deliberately selected to give similar proportions of academia versus industry as well as field of expertise, i.e. technical, marketing, social science, and legal. The questionnaire comprised over 30 questions covering mobile search trends with regard to technology, business models, market structure and privacy issues. A total of 54 replies were collected.

The survey results were scrutinised by the authors and prepared for the subsequent Delphi step, namely a face-to-face workshop to discuss the results of the first phase. For this purpose the "Exploring the Future of Mobile Search" workshop was organised in Ghent, Belgium, during the Conference of Telecommunication, Media and Internet Techno-Economics 2010 to convey different perspectives on trends and dynamics of mobile search. The panel was composed of 16 participants: policy experts; analysts from academia, consultancy companies and standardisation bodies; researchers with expertise in interaction design, user studies and mobile search technologies; and finally entrepreneurs and innovators who have built and are marketing new mobile search applications and services.

The workshop was organised in the following way. First each expert presented the aspect of mobile search that he or she considered the most important for shaping the future of the area. Then a round table was set up to discuss the experts' opinions with regard to the survey results and their opinions of the other experts' presentations. For the sake of clarity the discussion was structured around three topics:

(1) the need for mobile-adapted content and usage patterns of search "on the move";

(2) the evolution, bottlenecks and future challenges for the mobile search market; and

(3) Europe's position with regard to the rest of the world.

Unlike the traditional Delphi, method the aim of the discussion was not particularly geared to reach consensus on predictions, which were often mutually exclusive, but rather to foster a multi-stakeholder outlook on mobile search, pulling together different opinions to help build a shared understanding of the complex relations that are shaping the evolution of this area.

Next the main findings that emerged from the discussion - with the exception of the outcome on "the position of Europe" debate, which is not the subject of this paper - are presented. Those findings are contextualised against the existing scholarly literature. 
It is important to note, however, that much like the market itself, mobile search as an area of research is still in its infancy. In particular the market context is largely unexplored. To the authors' knowledge a report published by the European Commission (Gómez-Barroso et al., 2010), which forms the background to the workshop, is the only comprehensive public study on this subject. Consequently, instead of presenting a separate literature review - which would give an incomplete picture of mobile search market issues and trends - references to relevant previous work are embedded within the discussion. Note also that, at present, there are no reliable statistics to validate or disprove some of the perceptions of the experts.

\section{Mobile search specificity}

\section{Mobile search versus wired internet search}

How fundamentally is mobile search different from $\mathrm{PC}$ search? From a functional point of view search is about making the best use of available metadata to provide the user with meaningful information in spite of the fact that the user's request is often poorly formulated and typically unanticipated. The commonly accepted functional breakdown model of a generic search engine, which describes a search engine regardless of the application domain or business sector, thus applies also for mobile search. That the operation of a specific search engine can be traced back to a common conceptual model does not exclude major differences in technology, conditions and particularly usage (Westlund et al., 2011). Similar to enterprise search, which has major differences from intemet search (Bennett, 2008), this applies also to mobile search.

What are these distinctive features? For many of the experts the ability to search for locally relevant information (weather, news, traffic) is notably the most distinctive aspect of mobile search, while others see only an insignificant difference in the use of local search on the mobile phone with regard to PCs, and point out that it is instead the user expectations that make the difference. Despite nuances in such interpretations there is ample consensus that mobile search is taking an autonomous path beyond adaptation of the present web search to the mobile domain.

The main argument supporting this view is that the mobile is perceived as the most personal and confidential piece of all technological devices currently in use. Mobiles are all-day companions storing vast amounts of personal data, which are employed actively or passively across contexts and activities in a natural and dynamic way. They are used in a rush, while commuting, to fill idle time, while queuing, at home, or comfortably sitting on a sofa. The term "mobile" refers to its inherent specificity to be used "on the move", but the trend is also to use it in static environments. Many subscribers have already cancelled fixed telephone contracts and are using their mobiles in their homes. Moreover a number of mass applications, such as visiting social network sites, are increasingly accessed from mobile devices for simplicity (e.g. everywhere, anytime) and convenience (e.g. posting photos instantly), even at home (e.g. you do not have to switch on the computer; the mobile is always on). From a socio-economic perspective it is thus time to get rid of the assumption that mobiles are only used "on the move". Otherwise we risk missing out on important behavioural patterns with regard to when and how people seek answers, what they look for and how they make sense of different resources when interacting with their mobiles (Okazaki and Romero, 2010). Concluding it can be said that a differentiated view is needed to monitor and understand user patterns in different contextual environments; 
this requires more research involving context-dependent experiments (such as those of Liu et al., 2010; Lane et al., 2010).

\section{The future evolution of mobile search}

According to workshop experts current efforts in context-aware research are mainly focused on location-aware systems, i.e. aiming to enhance the search experience by making use of the user's location (Gronli and Ghinea, 2010). This will remain an important research domain in the medium term, because location is a kind of "first order proxy" for human activity and one of the very few parameters that are easy and cheap to capture and a relatively easy to handle filter for machine processing by a search engine. Other technologies requiring cognitive elements (e.g. mirroring how human cognition works) or semantic modules (e.g. how people make sense of reality) are largely immature particularly for a mobile context and/or too expensive for a mass product. When location relevance has become a basic building block, then it is often combined with other means so as to filter relevant information, such as by harnessing recommendations from the user's social network. Some proof of concept studies (Gui et al., 2009; Sun et al., 2009) are under way and commercial applications are also appearing.

Another contextual element on the rise is including augmented reality features by coating the external environment with a layer of information. Taking pictures of alpine landscapes with a mobile and receiving back the name and the height of peaks captured on the original picture is just one example of current research combining image search with geo-located data (Fröhlich et al., 2011).

A distinctive feature of many mobile search needs is its ephemeral nature. Experts subscribe to the view that searches may either:

- be evoked by a very urgent need (when is the next train to X leaving from where I am towards my home town?);

- be triggered by the desire to fill idle time (which is the funniest film on today?); or

- be prompted by an event, a situation, though there is no need to fulfil them on the spot (which cinema shows the film I have just seen the announcement about?).

The three aforementioned situations require different assets for successful retrieval. In the first case an appropriate combination of location-based filtering and semantic tools is helpful, while in the second case it is the combination of mobile-tailored content and social network tagging. In the third case the need is ephemeral, but the response is not. Decoupling the query from the analysis of the retrieved results is the new concept of applications that build upon a search inspiration concept like a kind of "post-it" memo for further action.

Mastering the social context is the economically most promising, technologically most demanding, and socially most useful dimension. It is even more so, if it exploits the mobile specificity (Wareham et al., 2009). Relatively simple concepts are being explored lately, for instance based on the claim that "wisdom of crowds" concepts can be introduced to reveal the intent and curiosity of individuals, i.e. the previous experience of other users can provide better suggestions and more appropriate advice (Church et al., 2010; Heiner and Balandin, 2007). Given the technological limitations, anticipation becomes a more important factor for mobile content search than for PC search. Curiosity is the main motivation for casual mobile search, whenever a user is 
not purposefully seeking a specific bit of information, but rather engaged in a playful exploration. Such a mobile content "discovery experience" could rely upon a physically instantiated set of queries and interactions that map other people's (prior) searches. The coating of mobile search with social networking capabilities would allow the user's friends to answer the user's queries while she/he is on the move.

A visionary long-term search concept may rely upon the future ability to capture, analyse and retrieve real-time continuous streams of information of different natures (environment, time of day, neighbouring mobile and other devices, location, friends nearby, etc). The assumption is that in the foreseeable future internet of things - all objects in the world seamlessly integrated into the information network - distributed sensor networks (spatially distributed autonomous sensors able to monitor physical or environmental conditions), "location-aware people" (permanently equipped with devices that can passively or actively determine their location) and other interoperable networks will be available. In such a scenario of vast data flows, there would be continually updated information relevant to the search query. As the information flows, queries will not be static but dynamic, as they may change as time goes by, as the user moves, as other actors get closer, etc.

As a last remark experts agree that in the realm of cloud computing via mobile internet access, search applications can become the indispensable bridge between personal content and usage contexts, allowing the user anytime anywhere to access relevant information.

\section{Barriers and challenges}

Market forecasts, the growing number of new services, and experts' opinions coherently indicate a bright future for mobile search. Nevertheless a number of uncertainties still remain about how the sector will evolve. Our analysis points to both technological and socio-economic factors.

\section{Technological factors}

The results of the survey previous to the workshop indicated technology to be only a minor barrier for the deployment of the mobile search applications, as 78 per cent of respondents claim that the main technological components - devices, networks, and applications - for mobile search are already available.

However development work is still badly needed: about half of the respondents stated that technical aspects make up a major barrier to the full deployment of mobile search services. Workshop experts confirm that - in spite of the general availability the technology is not yet mature and reliable enough for high performance in a mass user environment. Some enabling technology necessary for further boosting mobile search deployment - such as wireless networks, sensor networks, smart devices, and the cloud computing framework - are slowly entering the market, but not yet in a pervasive manner. Equally, current user tracking for geo-positioning is not sufficiently fine-grained for optimal service provision/fruition.

Technologies to enhance interaction need further improvement: gesture-based interfaces are not robust enough to sustain interaction in real-world environments, furthermore they are not as "natural" as they have been reported to be. Similarly, although many consider voice will become the natural way to interface in future mobile search applications and the obvious interface for illiterate users, voice recognition 
accuracy is only increasing very slowly. Voice-based interfaces are often disappointing from a user-experience point of view.

A warning should be noted: progress in a single technology is often not the winning approach in a complex mobile ecosystem where stakeholders are diverse and heterogeneous (Feijóo et al., 2009), including device manufacturers, mobile network operators, infrastructure providers, mobile OS providers, PC search players, and mobile-specific search players. Indeed no single technological advance or improvement in human-computer interaction is likely to have disruptive effects on mobile search take-up, even though we are observing important changes in the landscape due for instance to the penetration of touch-based interfaces (Ahmed et al., 2010; Seichter et al., 2009), which are shaping a new wave in the mobile content trajectory.

Linked to the previous point, standards have been identified as an enabling measure for encouraging the development of applications. While it is important to monitor the emergence of enabling technologies, the importance of standardisation cannot be underestimated, when projecting the potential penetration of cutting edge advancements. Considerable effort is being devoted by bodies such as the World Wide Web Consortium (W3C) to reach consensus around ontologies - both media ontology and context of delivery ontology - metadata formats that enhance discovery, timestamps for the web, interactions with social networks, speech synthesis or gesture-based interaction. However the experts all acknowledged that complex topics, such as context-awareness or privacy in relation to user personal identity data, need further research before a standardisation roadmap can be created.

\section{Socio-economic factors}

The majority of experts who completed the online questionnaire overwhelmingly believe the major challenges for mobile search are of an economic nature. This finding triggered the workshop discussion on business and revenue models for mobile search. The panel unanimously acknowledged one of the main bottlenecks for the development of the mobile search market to be the lack of widespread penetration of data flat rates and the absence of data roaming flat rates, or at least of predictable rates. Services are not going to spring up and flourish until the take-up of the mobile internet is promoted at access level. The promotion of Wi-Fi hotspots, not fully exploited at present, could be a driver.

Disruptive business models have not emerged yet. Some of the participants mentioned that an evolution of marketing promotion strategies is to be expected: advertising "as-we-know-it" is a concept that does not fit today's media (Okazaki and Barwise, 2011; Wilken and Sinclair, 2009). Advertising is the model that makes the most out of broadcasting communication channels. Mobile networks enable narrowcasting, potentially reaching the level of one-to-one marketing, allowing for a customised mix. Today's technology enables a paradigm shift in product/service promotion leading to a model where customer engagement is the main key to success. However solutions have to be tailored to the type of service as well as to the target audience (McClure, 2011; Kim and Han, 2009). Different business models are currently being explored with the objective to provide customers with a satisfactory search experience (Gómez-Barroso et al., 2011a). Here user satisfaction is going to be benchmarked against the speed, relevance, and usefulness of retrieved information. It is unlikely that a single winning revenue model will emerge fitting all business models. 
From the user perspective a matter of particular concern is the availability of personal information for service provision and the related privacy issue. Anticipatory functions, such as serendipitous content discovery, would require a higher degree of user profiling and behavioural tracking than we are currently accustomed to. This causes a dilemma. On the one hand extended access to personal data provokes privacy concerns (Zhou, 2011; Gurau and Ranchhod, 2009; Castañeda et al., 2007). On the other hand the lack of personalised data limits the value of useful applications. Moreover a privacy backlash could prejudice people against advanced mobile search. The panel did not reach a consensus on the formulation of the problem and its implications.

\section{Conclusions}

The main factors shaping the evolution of this segment of the mobile sector are multiple. The main messages that can be drawn from this qualitative exercise are that there is no consensus on what mobile search is about in terms of both user needs and mobile specific search requirements. Mobile human-computer interaction is a young domain that needs further support so as to drive innovation in the field. There are still many unknowns concerning the specificity of search behaviours coupled with mobile technology and they require in-depth research.

Experts share a great deal of consensus when asked about technical constraints, (e.g. screen size or information input aspects) and technical opportunities (e.g. taking advantage of sensing contextual information). Consensus, though, diminishes when it comes to the key aspects of mobile search. For some the ability to search for locally relevant information represents the most distinctive aspect of mobile search. For others there is no significant difference between local search on the mobile phone and on a PC. Some mentioned that mobile search is about "fresh" content; others claimed that mobile users search for "snacks of content", or for very concrete results. While some experts claimed that socially enhanced search is the real asset and emerging trend, others argued that, with the mobile being the remote control of our lives, mobile search is much more about finding personal content. Some of the participants challenged the discussion by asserting that the mobile should be considered as a new media platform using its own content ecosystem, thus requiring a completely different approach to search. The differences in opinion suggest that we are still focusing on the individual pieces of the puzzle, far from being able to sketch the overall picture.

It was observed that one of the reasons for the coexistence of so many different viewpoints can be revealed by adopting a historical perspective: the mobile web builds on the legacy of the "walled garden" characterisation of mobile telecommunication (Ramos et al., 2009). Mobile network operators and content aggregators have been shaping access to content based on a portal model with associated revenue models, while innovative business models are struggling to consolidate. Experts agree on that, since search applications are built by the integration of different technological bricks, by the mash-up of content and applications, and lever on social networks to harness recommendations from collective search and rating behaviours. Hence the promotion of an open ecosystem is a critical boosting factor.

Finally it was unanimously acknowledged that the infrastructure has to become more pervasive: broadband connections constitute the main enabler of mobile search, and need pushing (both in terms of availability and affordability) so as to foster the creation and take-up of innovative services. 
Further steps

The potential of mobile search remains largely untapped. Moreover a genuinely-adapted-to-mobile search is a piece of the puzzle for fully exploiting the potential of smartphones, tablets and other portable devices to which an increasing number of people have access.

This paper reports on an exploratory study that could serve as a basis for further research in the field. To this end the following issues, grouped around three broad topics, could - and need to - be addressed to take the research forward:

(1) Technologies driving the evolution of mobile search will continue progressing. Leaving aside the research on technical aspects, efforts aimed towards getting new inventions to work will undoubtedly be required. That would include the focusing of those developments toward specific services as well as all stages of the exploitation process and commercial activities.

(2) On the demand side, adoption and use of mobile search offer huge potential for research. Improving knowledge on information retrieval and information seeking, motives and information needs is key to developing new applications and services. Analyses of large-scale logs from search engine databases is the most common approach but experimental research - group studies, living labs - can deliver remarkable results.

(3) On the supply side, the dynamics of the market need to be addressed. Business models and revenue schemes are the most obvious candidates for a thorough analysis. The economic value of the search functionality with regard to the totality of the value chain of the mobile services is another promising direction for research.

\section{References}

Ahmed, F., Islam, M.A., Borodin, Y. and Ramakrishnan, I.V. (2010), "Assistive web browsing with touch interfaces", Proceedings of the 12th International ACM SIGACCESS Conference on Computers and Accessibility, ACM Press, New York, NY, pp. 235-6.

Bennett, M. (2008), "20+ differences between internet vs enterprise search - and why you should care", New Idea Engineering, Inc. Newsletter, Vol. 5 Nos 2-4, available at: www.ideaeng. com/tabId/98/itemId/154/20-Differences-Between-Internet-vs-Enterprise-Se.aspx (accessed 15 May 2011).

Castañeda, J.A., Montoso, F.J. and Luque, T. (2007), "The dimensionality of customer privacy concern on the internet", Online Information Review, Vol. 31 No. 4, pp. 420-39.

Church, K., Neumann, J., Cherubini, M. and Oliver, N. (2010), "SocialSearchBrowser: a novel mobile search and information discovery tool", Proceedings of the 14th ACM International Conference on Intelligent User Interfaces, ACM Press, New York, NY, pp. 101-10.

Feijoo, C., Maghiros, I., Abadie, F. and Gómez-Barroso, J.L. (2009), "Exploring a heterogeneous and fragmented digital ecosystem: mobile content", Telematics and Informatics, Vol. 26 No. 3, pp. 282-92.

Fröhlich, P., Oulasvirta, A., Baldauf, M. and Nurminen, A. (2011), "On the move, wirelessly connected to the world", Communications of the ACM, Vol. 54 No. 1, pp. 132-8.

Gartner (2010), "Gartner says worldwide mobile phone sales grew 35 percent in third quarter 2010; smartphone sales increased 96 percent", Gartner Newsroom, 10 November, available at: www.gartner.com/it/page.jsp?id=1466313 (accessed 15 May 2011). 
Gómez-Barroso, J.L., Feijóo, C. and Compañó, R. (2011a), "Promising prospects in mobile search: business-as-usual or techno-economic disruptions?", IEEE Signal Processing Magazine, Vol. 28 No. 4, pp. 131-5.

Gómez-Barroso, J.L., Feijóo, C. and Compaño, R. (2011b), "Opportunities in the mobile search market", Computer, Vol. 44 No. 11, pp. 93-5.

Gómez-Barroso, J.L., Compañó, R., Feijōo, C., Bacigalupo, M., Westlund, O., Ramos, S., Jaokar, A., Álvarez, F., De Waele, R., Mateos-Barrado, G. and García-Jiménez, M.C. (2010), "Prospects of mobile search", Document EUR 24148 EN, Publications Office of the European Union, Luxembourg, available at: http://ipts.jrc.ec.europa.eu/publications/pub.cfm?id=3159 (accessed 15 May 2011).

Grønli, T.M. and Ghinea, G. (2010), "Three-dimensional context-aware tailoring of information", Online Information Review, Vol. 34 No. 6, pp. 892-906.

Gui, F., Adjouadi, M. and Rishe, N. (2009), “A contextualized and personalized approach for mobile search", Proceedings of the 23rd International Conference on Advanced Information Networking and Applications Workshops, IEEE Computer Society Press, Los Alamitos, CA, pp. 966-71.

Gurau, C. and Ranchhod, A. (2009), "Consumer privacy issues in mobile commerce: a comparative study of British, French and Romanian consumers", Joumal of Consumer Marketing, Vol. 26 No. 7, pp. 496-507.

Heiner, A. and Balandin, S. (2007), "Social search for efficient information location in mobile domains", Proceedings of the 4th IEEE Consumer Communications and Networking Conference, IEEE Computer Society Press, Los Alamitos, CA, pp. 1172-3.

Kim, B. and Han, I. (2009), "What drives the adoption of mobile data services? An approach from a value perspective", Joumal of Information Technology, Vol. 24 No. 1, pp. 35-45.

Lane, N.D., Lymberopoulos, D., Zhao, F. and Campbell, A.T. (2010), "Hapori: context-based local search for mobile phones using community behavioral modeling and similarity", Proceedings of the 2010 ACM Conference on Ubiquitous Computing, ACM Press, New York, NY, pp. 109-18.

Liu, C.Y., Rau, P.L.P. and Gao, F. (2010), "Mobile information search for location-based information”, Computers in Industry, Vol. 61 No. 4, pp. 364-71.

McClure, M. (2011), “Creating a recipe for success: mobile search in action”, EContent, Vol. 34 No. 4 , pp. $22-6$.

Mixi (2010), "Number of Mixi smartphone users tops one million”, Japan's Cellphone Edge \& Beyond, 6 November, available at: www.analytica1st.com/2010/11/number-of-mixismartphone-users-tops.html (accessed 15 May 2011).

Nielsen (2011), "Apple leads smartphone race, while Android attracts most recent customers", NielsenWire, 3 January, available at: http://blog.nielsen.com/nielsenwire/online_mobile/ apple-leads-smartphone-race-while-android-attracts-most-recent-customers (accessed 15 May 2011).

Okazaki, S. and Barwise, P. (2011), "Has the time finally come for the medium of the future? Research on mobile advertising", Journal of Advertising Research, Vol. 51 No. 1, pp. 59-71.

Okazaki, S. and Romero, J. (2010), "Online media rivalry: a latent class model for mobile and PC internet users", Online Information Review, Vol. 34 No. 1, pp. 98-114.

Organisation for Economic Co-operation and Development (2010), "OECD broadband statistics June 2010", available at: www.oecd.org/sti/ict/broadband (accessed 15 May 2011). 
Ramos, S., Feijóo, C. and Gómez-Barroso, J.L. (2009), "Next generation mobile network deployment strategies", Journal of the Institute of Telecommunications Professionals, Vol. 3 No. 1, pp. 13-19.

Seichter, H., Grasset, R., Looser, J. and Billinghurst, M. (2009), "Multitouch interaction for tangible user interfaces", in Klinker, G., Saito, H. and Hollerer, T. (Eds), Proceedings of the 8th IEEE International Symposium on Mixed and Augmented Reality, IEEE Computer Society Press, Los Alamitos, CA, pp. 213-4.

Sun, J., Koong, K.S. and Poole, M.S. (2009), "Critical success factors for context-aware mobile communication systems", International Journal of Mobile Communications, Vol. 7 No. 3 , pp. 290-307.

Wareham, J.D., Busquets, X. and Austin, R.D. (2009), "Creative, convergent, and social: prospects for mobile computing", Journal of Information Technology, Vol. 24 No. 2, pp. 139-43.

Westlund, O., Gómez-Barroso, J.L., Compañó, R. and Feijóo, C. (2011), "Exploring the logic of mobile search", Behaviour \& Information Technology, Vol. 30 No. 5, pp. 691-703.

Wilken, R. and Sinclair, J. (2009), "Waiting for the kiss of life: mobile media and advertising", Convergence, Vol. 15 No. 4, pp. 427-45.

Zhou, T. (2011), "The impact of privacy concern on user adoption of location-based services", Industrial Management \& Data Systems, Vol. 111 No. 2, pp. 212-26.

\section{Appendix}

The results of the online questionnaire that preceded the workshop "Exploring the Future of Mobile Search" are available at: http://is.jrc.ec.europa.eu/pages/TFS/documents/IPTS-MobileSearch-Survey-2010-Results-WEB.pdf

The programme and presentations made at the workshop are available at: http:/is.jrc.ec. europa.eu/pages/TFS/searchworkshop.html

\section{About the authors}

José Luis Gómez-Barroso is with the Universidad Nacional de Educación a Distancia, Spain (UNED). He holds a Master's degree and a $\mathrm{PhD}$ in Economics from UNED. He also received a Master's degree in Telecommunication Engineering from the Universidad Politécnica de Madrid, as well as another in Law from the Universidad Complutense. His teaching and research interests are in the area of economics and regulation of the telecommunications industry and public aspects of the development of information society. José Luis Gómez-Barroso is the corresponding author and can be contacted at: jlgomez@cee.uned.es

Margherita Bacigalupo holds a $\mathrm{PhD}$ in Telematics and Information Society from the Department of Electronic Engineering of the University of Florence and a BSc + MSc in Communication Science obtained fron the University of Siena. She has a broad experience in interaction design ranging from the design and evaluation of robotic artifacts in the context of dementia care to the design of innovative concepts for future air traffic management scenarios. She worked as a Scientific Officer at the Institute for Prospective Technological Studies of the Joint Research Centre until early 2011. In this position she contributed to the Institute's research activity on electronic identity whereas in the area of mobile content evolution she worked on future trends in the mobile search market.

Stavri G. Nikolov is a Senior Scientist at European Commission's Institute for Prospective Technological Studies (JRC-IPTS), doing research in the areas of digital identity, eID technologies, eID architectures, and mobile and enterprise search. Previously, he was Managing Director and Head of Research of Attentive Displays Ltd, a start-up company which he co-founded in 2007. From 1998 until 2007, Dr Nikolov was a Research Fellow/Senior Research Fellow in Image Processing at the University of Bristol (UK). He is the creator and co-ordinator of 
The Online Resource for Research in Image Fusion and The Online Archive of Scanpath Data as well as the Founding Director of the Digital Spaces Living Lab in Sofia, Bulgaria, focussed on novel digital media technologies.

Ramón Compañó is the Programme Manager of the Institute for Prospective Technological Studies (Joint Research Centre of the European Commission). He graduated in Physics from the Technical University of Aachen, and completed a $\mathrm{PhD}$ at both Aachen and the University of Modena in Italy. He also holds a Master's degree in Technology Management from Solvay/ULB in Brussels. Since he joined the European Commission in 1993 he served as project manager in different R\&D domains including standardisation, advanced materials, physical analysis, including surface science, metrology and micro/nano-electronics. In 2004, he joined the Institute for Prospective Technological Studies, where he analyses the impacts of emerging technologies on society and economy.

Claudio Feijoo is Professor at Universidad Politécnica de Madrid, where he researches on the future socio-economic impact of emerging information society technologies, in particular, from a mobile and/or content perspective. From his work experience, he enjoyed very much his two years at the Institute for Prospective Technological Studies of the European Commission, the direction of the Chair in Telecommunications Regulation and Information Society Public Policies at Universidad Politécnica de Madrid, and participating in information society development plans while being adviser to the Spanish State Secretary on telecommunications and the information society. 